\title{
Maximum Likelihood Estimation of Doppler Centroid in Synthetic Aperture Radar Images
}

\author{
Pietro Guccione \\ Electrical and Information Engineering Department, Politecnico di Bari, Bari 70125, Italy
}

\begin{abstract}
This paper addresses the problem of estimating the DC (doppler centroid) for SAR (synthetic aperture radar) data, in presence of speckle and thermal noise. The main idea is to exploit a bandwidth much wider than the PRF (pulse repetition frequency), say 3-5 times, by exploiting strong point targets. Natural and isolated targets with close-to-ideal features are focused using a digital spotlight algorithm applied to stripmap acquisitions; then, accurate DC is estimated as the relative shift of the targets azimuth spectra using the ML (maximum likelihood). The Cramer-Rao bound of the estimate is shown to be lower than that of the conventional estimators based on traditional stripmap focusing of $Q+1$ times for high SNR (signal-to-noise ratio) of targets, being $Q$ the oversampling factor of the spotlight processing compared to the stripmap one. The method is applied to both simulated targets and a real dataset coming from the Cosmo SkyMed X-band constellation.
\end{abstract}

Key words: SAR, DC, ML estimation, spotlight processing.

\section{Introduction}

Estimation of the Doppler frequency shift is a fundamental issue in the radar data processing: Both weather radar and moving-target indication radar are based on this principle [1-2]. In SAR (synthetic aperture radar) processing the accurate determination of the target Doppler history is used to perform a correct azimuth compression. The mean of the Doppler frequency, i.e., the DC (doppler centroid), is in fact a measure of the antenna squint. Lacking of accuracy in determination of DC results in a radiometric degradation and possibly a loss in signal-to-ambiguity ratio since the centroid is used to center the bandpass azimuth compression filter to the effective location of the spectrum.

Estimation of DC from data is not new in literature and has been solved some decades ago [3-6]. However, the problem is always up-to-date because of its particular relevance in accurate location of targets, especially for a new generation of large bandwidth

Corresponding author: Pietro Guccione, Ph.D., assistant professor, research fields: signal processing and remote sensing. E-mail: pietro.guccione@poliba.it. sensors. The estimation of DC is usually intended as both the ambiguous estimation (within the PRF (pulse repetition frequency)) and the ambiguity number (i.e., the integer part of the PRF). Ambiguity is usually estimated without error by geometry or by exploiting ad-hoc techniques [7-9]. Ambiguous estimation of DC, instead, is traditionally based on correlation techniques.

The estimation accuracy is highly dependent on the number of used independent samples and on the homogeneity of the reflectivity of the selected region. The effective number of independent samples is, in fact, decreased by the scene contrast factor, i.e., the mean of the scene divided by its standard deviation. The difficulty of achieve homogeneous scenes for every acquisition, especially in urban or coastal areas, makes often the estimation unreliable. On the other hand, a geometric approach based on the knowledge of sensor attitude and orbit, is good enough to track the Doppler ambiguity but not to provide an accurate DC value. Effective current DC estimation algorithms suffer from lacking of accuracy proportional to the sampling frequency that results in an unreliable estimation of up to $5 \%$ of the PRF. This quantity 
amounts to about $150 \mathrm{~Hz}$ and $80 \mathrm{~Hz}$ for $\mathrm{X}$ - and C-band sensors, respectively.

The key idea of this paper is to provide an accurate estimate of the Doppler Centroid as shift from zero of the azimuth spectrum of close-to-ideal point targets. Point-like targets are indeed minimally affected by alias since their azimuth spectrum has sidelobes stronger than the average background. Estimation of not-aliased azimuth spectrum using natural ideal targets has been originally proposed in Ref. [10]. Here, the idea is extended using digital spotlight focusing on stripmap data [11] and limiting the processing to strong and isolated targets, in order to reduce the effect of alias of nearby clutter [12-13]. The DC, representing the shift with respect to zero of the azimuth spectrum, is then estimated using the ML (maximum likelihood), reducing this way the risk of statistics fluctuation due to other causes (the azimuth spectra are modified also by the target real size, shape and the area offered to the radar illumination).

The final accuracy is a function of the number of targets involved in the estimate and of their SNR (signal-to-noise ratio). In detail, it is shown that the RMSE (root mean square error) of the estimate approaches to the theoretical CRB (Cramer-Rao bound) for targets with moderate SNR, let's say $\sim 15 \mathrm{~dB}$. This result outperforms any other correlation-based estimation method, for which homogeneous backscatterer and stripmap processing are usually assumed.

The remainder of the paper is organized as follows. In Section 2 the model of acquisition and processing SAR data is given; in Section 3 the problem of estimating the DC is faced, formulating the problem for a single and then for many targets and giving also the CRB of the ML estimate. In Section 4 the experimental results using both simulated and real data are presented and discussed; finally in Section 5 some conclusions are drawn.

\section{The Data Model}

The continuous-time SAR acquisition and focusing system is represented in Fig. 1. In the following:

$\gamma_{o}(t, r)$ is the complex reflectivity of a distributed and homogeneous scatterer, which is regarded as a realization of a complex, spatially uncorrelated zero-mean Gaussian process with variance $\sigma_{s}^{2}$ (fully-developed speckle).

$p(t, r)=a \cdot \delta(t, r) \quad$ is an ideal point target superimposed to the distributed scatterer. This ideal assumption is relaxed successively.

$n_{t h}(t, r)$ is the received thermal noise, superimposed to data. The received noise is assumed to be a stationary white, circular complex zero-mean Gaussian process with variance $\sigma_{n}^{2}$.

In this simplified formulation the variation of the Doppler with range frequency is neglected; since the fractional bandwidth is usually small in SAR, the
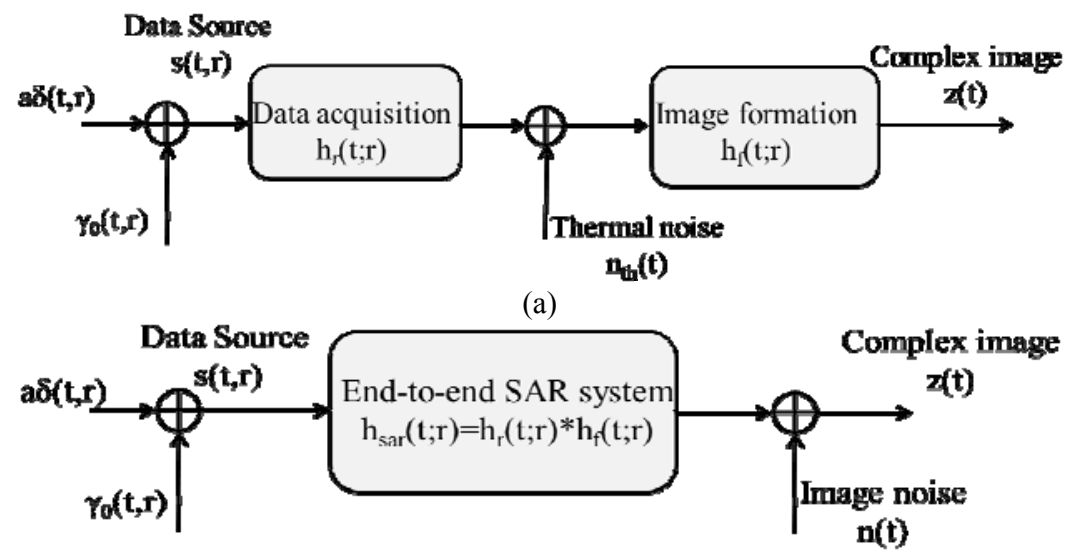

(b)

Fig. 1 The continuous-time SAR system in the slow time, $t$. (a) the acquisition and image formation processes; (b) the equivalent (and simplified) end-to-end system. 
analysis is limited to the mono-dimensional case.

The simplified model for image acquisition (Fig. 1a) can be regarded as a linear, space-variant filter $[5,13]$ :

$$
h_{r}(t ; r) \simeq A_{a n t}\left(\frac{v}{r}\left(t-t_{d c}\right)\right) \cdot \exp \left(j \pi f_{R} t^{2}\right)
$$

where $\psi_{d c}=v t_{d c} / r$ is the squint angle due to the centroid, $t_{d c}$ is the azimuth time where the Doppler frequency equals the centroid, $r$ is the slant range of the target, $v$ is the sensor speed, $f_{R}=-2 v^{2} / \lambda r$ the Doppler rate and $A_{a n t}(t ; r)$ is the azimuth antenna pattern. Focusing (or image formation) is the process that regresses raw data back to an estimate of the complex reflectivity. The commonly used approach to perform focusing is to build a phase matched filtering $h_{f}(t ; r)$ such that: $\angle h_{f}(t ; r)=\exp \left(-j \pi f_{R} t^{2}\right)$.

The cascade of acquisition and focusing represents the end-to-end SAR system and can be regarded as a linear time-variant process that achieves the image data when the input is the complex reflectivity (see the equivalent scheme in Fig. 1b):

$$
z(t)=h_{s a r}(t ; r) * \mathrm{~s}(\mathrm{t} ; \mathrm{r})+\mathrm{n}(\mathrm{t})
$$

where we have: $h_{s a r}(t ; r)=h_{r}(t ; r) * h_{f}(t ; r) \quad$ and $s(t, r)=\gamma_{o}(t, r)+\mathrm{p}(\mathrm{t}, \mathrm{r})$. According to the bandwidth of the focusing operator, in the equivalent end-to-end model the additive image noise: $n(t)=n_{t h}(t) * h_{f}(t ; r)$ is still Gaussian zero-mean but no longer a white process.

\section{Doppler Centroid Estimation}

According to details in Appendix 1, the azimuth spectrum of the end-to-end SAR system (and in particular, the position of the maximum of the antenna) is function of DC. The main idea applied in this paper is to collect such spectra for many point-like targets in order to perform a statistical estimate of the centroid.

Alias between targets occurs because of the sampled nature of the image acquisition along azimuth. The effect of alias is different on a point target and on the distributed scatterers. For point targets the spectral replica are separated by PRF in the frequency domain (which corresponds to $\Delta T=\mathrm{PRF} / f_{R}$ in the time domain). This spectral separation allows targets to be processed using a Spotlight kernel [14] without aliasing. The same processing cannot be applied, however, to distributed scatterers as summing occurs at different frequencies, providing a zero-mean normal and uncorrelated process (details are in Ref. [15]).

\subsection{Single Target Statistical Model and ML Estimation}

Let us formulate the problem in a discrete context for a single target.

Under the hypothesis of multivariate Gaussian process (reasonable for SAR data) the second order moment of $z(t)$ represents a sufficient statistics to infer information from data. Let us discretize the azimuth non-ambiguous interval $[-Q \cdot P R F / 2, Q \cdot P R F / 2]$ in $M$ samples ( $Q$ is the spotlight oversampling integer factor with respect to the nominal stripmap sampling frequency). The sampled model of Eq. (2) can be rewritten using the vector notation:

$$
\begin{aligned}
& \mathbf{z}=[z(1), \ldots, z(M)]^{T}=\mathbf{h s}+\mathbf{n} \\
& \mathbf{s}=\boldsymbol{\gamma}_{\mathbf{o}}+\mathbf{p}
\end{aligned}
$$

being $\mathbf{n}$ the N-column array of the image noise $n(t)$ sampled in the azimuth direction (the number of samples in the azimuth Doppler history, $\mathrm{N}$, is supposed larger than $\mathrm{M}), \boldsymbol{\gamma}_{o}$ and $\mathbf{p}$ the N-column arrays of the distributed and point target sources and $\mathbf{h}$ the (approximately) circulant matrix of size $M \times N$ that can be generated from the sampled version of $h_{\text {sar }}$ to represent the convolution by a matrix-vector multiplication. Finally, $[\bullet]^{T}$ is the transpose operator. After few passages (details are in Appendix), the covariance matrix can be written as:

$$
\mathbf{C} \simeq \operatorname{diag}\left[\sigma_{s}^{2}\left|H\left(f_{m}\right)\right|^{2}+\sigma_{n}^{2}\right], \quad m=1, \ldots, M
$$

The model assumed for the DC is $f_{d c}=\tilde{f}_{d c}+v$ 
being $v$ the unknown residual DC, i.e., the parameter to be estimated after a first, raw estimation $\tilde{f}_{d c}$ based on conventional methods or on satellite geometrical parameters. After a shift of $\tilde{f}_{d c}$, the power spectral density of $\mathbf{z}, S_{z}(f)$, is a function of $v$ only: $S_{z}(f) \simeq|S(f)|^{2} A_{a n t}^{2}(f-v)$ (from Eq. (A2)). Since the process achieved by transforming $\mathbf{z}$ is still multivariate normal distributed, the LLH ( $\log$-likelihood) of the parameter $v$, known $\mathbf{Z}$, has a closed expression:

$$
\ell(v \mid \mathbf{Z})=-\frac{M}{2}\left[M \log (2 \pi)+\mathbf{Z}^{*} \mathbf{C}^{-1} \mathbf{Z}+\log \mathbf{C}\right]
$$

After some maths, a simple formulation for the optimal estimate of $v$ turns out:

$$
\hat{v}=\arg \max _{\nu}\left\{-\sum_{m=1}^{M} \frac{|Z(m)|^{2}}{\sigma_{s}^{2} A_{\text {ant }}^{2}\left(f_{m}-v\right)+\sigma_{n}^{2}}\right\}
$$

having used for $\mathbf{C}$ the approximation in Eq. (4). The optimal value for the residual $v$ can hence be explained as the value that maximizes the power of the scatterer whitened spectrum.

\subsection{Multi-Target Model and ML Estimation}

Now, we extend the previous model including more targets. The azimuth spectrum given in Eq. (A1), for $P$ targets is:

$$
\begin{aligned}
& Z_{p}(f)=A(f-v)\left|S_{p}(f)\right| \cdot \exp \left(-j \phi_{p}\left(f ; f_{d c}\right)\right) \\
& +N_{p}(f), \quad \mathrm{p}=1, \ldots, \mathrm{P}
\end{aligned}
$$

Here the residual DC $v$ is assumed approximately constant throughout the swath and can be assumed as the average inaccuracy in the estimation of centroid over the image.

For each frequency in the interval $f_{m} \in[-Q \cdot \mathrm{PRF} / 2, Q \cdot \mathrm{PRF} / 2]$, (7) can be arranged in a vector notation:

$$
\mathbf{Z}=\mathbf{X}+\mathbf{N}
$$

being $\mathbf{Z}$ the column vector of size $[P, 1]$ including the $Z_{p}\left(f_{m}\right), p=1, \ldots, P$ and:

$$
\mathbf{X}=A\left(f_{m}-v\right) \cdot(\mathbf{S} \circ \mathbf{\Phi})
$$

with $\mathbf{S}, \boldsymbol{\Phi}$ and $\mathbf{N}$ the vectorization of the terms in Eq. (7) for $f=f_{m}$ and $\circ$ the Hadamard (element-byelement) product.

The likelihood of the parameters $v$ and $\mathbf{S}$ provided the data $\mathbf{Z}, \forall f_{m}$, should account for the different target spectral shapes, $S_{p}(f)$ at the different frequencies. However, this is a difficult problem to treat due to the high number of unknowns. A simpler, even if approximate, formulation can be provided using targets with high SNR, for which $\angle Z_{p}(f) \simeq \phi_{p}, \forall f$. In that case the LLH of $v$ and $\mathbf{S}$ provided $\mathbf{Z}$ and $f=f_{m}$ boils down to:

$$
\begin{aligned}
& \ell\left(\mathbf{S}, v \mid \mathbf{Z}, f_{m}\right)= \\
& -\sum_{p=1}^{P}\left\{\frac{1}{\sigma_{p}^{2}}\left[\left|Z_{p}\right|-A\left(f_{m}-v\right) S_{p}\left(f_{m}\right)\right]^{2}+\log \sigma_{p}^{2}\right\}
\end{aligned}
$$

Maximizing Eq. (10) with respect to $\{\mathbf{S}, v\}$ means to get:

$$
\{\hat{\mathbf{S}}, \hat{v}\}=\arg \min _{S_{p}, \nu}\left\{\sum_{p=1}^{P} \frac{1}{\sigma_{p}^{2}}\left[\left|Z_{p}\right|-A\left(f_{m}-v\right) S_{p}\left(f_{m}\right)\right]^{2}\right\}
$$

In Eq. (11) a sum of square is present. Minimizing a sum of squares means to minimize separately each term. In a first approximation then the log-likelihood, provided $f=f_{m}$, can be approximated by the sum of the various log-likelihoods, although both the parameters $\left\{\mathrm{S}_{p}, v\right\}$ should be estimated. In principle, if all the targets were delta-like, the log-likelihood would be optimized just with respect to $v$, as $S_{p}(f)=1, \forall p, f_{m}$. A simpler solution consists in leaving the likelihood formulation as in Eq. (5), but changing the sum of log-likelihoods with a weighted sum, the weights quantifying the targets point-shape trend. From Eq. (11) it turns out clear to define such weights as inversely proportional to the energy of their difference (measured in the frequency domain) with respect to an ideal point target:

$$
\hat{I}_{p}=\left[\sqrt{\int\left|Z_{p}(f)-A_{i d}(f)\right|^{2} d f}\right]^{-1}
$$

where $A_{i d}$ is the folded version of the ideal antenna pattern shape. This quantity has been already defined 
in Ref. [17] and called similarity index of point targets. Intuitively, the higher the similarity of a target azimuth spectrum to the ideal (point-wise) azimuth spectrum, the higher is the index and so the higher is its weight in the formulation of the likelihood problem.

The multi-target log-likelihood of $v$, provided $\mathbf{Z}$ for all the targets is then a weighted average:

$$
\ell_{t o t}(v \mid \mathbf{Z}) \simeq \sum_{p=1}^{P} I_{p} \ell_{p}(v \mid \mathbf{Z}) / \sum_{p=1}^{P} I_{p}
$$

which provides a multi-target estimate of $v$ equals to:

$$
\hat{v}=\arg \max _{v}\left\{-\frac{1}{\sum_{p} I_{p}} \sum_{p=1}^{p} \sum_{m=1}^{M} I_{p} \cdot \frac{|Z(m, p)|^{2}}{\sigma_{s}^{2} A_{a n t}^{2}\left(f_{m}-v\right)+\sigma_{n}^{2}}\right\}
$$

It is here remarked that the solution proposed in Eq. (14) is approximate and does not accomplish for the target spectral shape $\mathbf{S}$. For this reason, the selection of target set should be limited to the most point-like, basing the screening process on the similarity of the spectrum with the ideal antenna model.

\subsection{Cramer-Rao Bound}

For circular complex Gaussian processes the CRB of an unknown (but deterministic) parameter can be found by numerical computation since it assumes a simple analytical form:

$$
\sigma_{c r b}^{2}=-\operatorname{tr}\left(\frac{\partial \mathbf{C}}{\partial v} \frac{\partial \mathbf{C}^{-1}}{\partial v}\right)^{-1}=\left\{\sum_{m}\left(\frac{S_{z}{ }^{\prime}\left(f_{m}\right)}{S_{z}\left(f_{m}\right)}\right)^{2}\right\}^{-1}
$$

being $\operatorname{tr}$ is the trace of the matrix and being $S_{z}(f)$ the diagonal elements of the covariance matrix $\mathbf{C}$. Eq. (15) shows that the most relevant spectral contributions in the CRB are the terms with high derivative and low power, i.e., the spectral parts close to the nulls; consequently, the estimate improves by using spectra that include sidelobes, i.e., spectra of targets focused using the digital spotlight processing. Numerical solution of Eq. (15) can be found by accounting for the level of aliasing, accordingly to the degree of oversampling (usually this is $3 \times$ or $5 \times$ the stripmap sampling frequency, PRF). Numerical solution suggests that the CRB is basically independent on the thermal noise level, as demonstrated in the simulations (see the dotted lines in Fig. 4).

To solve for the CRB asymptotic performance in the stripmap case, the aliased antenna spectrum $A(f)$ can be approximated by the first-order term of its Fourier series:

$$
A(f)=1+m \cdot \cos (2 \pi f / \mathrm{PRF})
$$

With $m=0.7$, which fits well for the usual level of aliasing (the azimuth bandwidth is $\simeq 0.8 \cdot \mathrm{PRF}$ for typical SAR systems), the CRB is $\sigma_{c r b} \simeq 0.22 \cdot \mathrm{PRF} / \sqrt{N}$, with $N$ the number of samples in the azimuth Doppler history. In the digital spotlight case it is found by numerical integration of Eq. (15) that the CRB for high SNR eventually approaches to $\sigma_{c r b} /(Q+1)$.

\section{Results on Simulated and Real Data}

The proposed estimator has been tested with both simulated and real datasets. A data stack of 25 Cosmo SkyMed images acquired on the same area has been considered. The acquisition parameters are summarized in Table 1; the same parameters have been used for the simulation. To simulate the targets spectral shape, i.e., their electromagnetic behavior as a function of the relative sensor position, a low-pass filter, modeled by a second-order polynomial, has been implemented tuning the low-pass amount by a Gaussian distributed random variable. Moreover, in the simulations, a not null uncertainty in DC, $\bar{v}$, has been applied; this value has been intentionally not corrected in the spotlight focusing, in order to simulate our lacking in the knowledge of the true $f_{d c}$. Estimation of unknown $\bar{v}$ has been performed by solving Eq. (14) in an exhaustive way and varying the research in a suitable interval.

One of the key steps of the algorithm is expressed 
Table 1 Acquisition parameters for Cosmo SkyMed and simulated datasets.

\begin{tabular}{|c|c|c|}
\hline Name & Value & UoM \\
\hline Carrier frequency & 9.60 & $\mathrm{GHz}$ \\
\hline Antenna length $\mathrm{L}_{\text {ant }}$ & 5.6 & $\mathrm{~m}$ \\
\hline Satellite mean speed $v_{\text {med }}$ & 7,627 & $\mathrm{~m} / \mathrm{s}$ \\
\hline Off-nadir ref angle & 26.55 & deg \\
\hline PRF & 3,056 & $\mathrm{~Hz}$ \\
\hline Pulse length & 40 & $\mu \mathrm{s}$ \\
\hline Bandwidth & 119.824 & $\mathrm{MHz}$ \\
\hline Sampling Frequency & 146.25 & $\mathrm{MHz}$ \\
\hline Sampling Window Length & 172.417 & $\mu \mathrm{s}$ \\
\hline Simulated SNR & {$[7 \div 50]$} & $\mathrm{dB}$ \\
\hline Antenna pointing & Right, yaw steered & \\
\hline Antenna aperture, $\mathrm{N}_{\text {samp }}$ & 2008 & Samples \\
\hline Orbit propagator & NORAD4 & \\
\hline Number of simulated aperture & $1,3,5$ & \\
\hline Simulated $\bar{v}$ & 10 & $\mathrm{~Hz}$ \\
\hline Number of simulated targets & 64 & \\
\hline Number of trials in simulation & 32 & \\
\hline
\end{tabular}

by the approximation in (A3), i.e., the hypothesis that the covariance matrix of the observations in frequency is basically diagonal, despite of the (band limited) clutter and noise.

Such approximation has been tested for various levels of average SNR of the simulated targets. In Fig. $2 \mathrm{~b}$ the sample covariance matrix achieved by using 128 simulated targets with an average $\mathrm{SNR}=15 \mathrm{~dB}$ is compared to the ideal model of (A3) (in Fig. 2a). As can be seen, the two matrices are visually indistinguishable and better results are obtained with higher SNR.

A good estimation of the covariance matrix is then the main hindrance for a good estimation of the DC. In principle, the covariance matrix would be better defined through the average of a large amount of spectra but, in practice, this solution provides a noisy estimate if targets with low SNR are included in the measure. In case of targets with low SNR better results are expected by using the square of the ideal antenna model (a folded $\operatorname{sinc}^{2}$ ) rather than the diagonal of the sample covariance matrix.

In Fig. 3 the RMSE of the DC estimates for the simulated dataset is represented as a function of the targets SNR. In one case the average of the targets spectra has been used for $A_{a n t}(\cdot)$ in the denominator of the expression in Eq. (14); in the other case the ideal antenna model has been used.

Comparison of the two curves suggests that $15 \mathrm{~dB}$ is a suitable threshold for the targets SNR under which the antenna model should be used in the denominator of Eq. (14) in place of the diagonal of the sample covariance matrix.

Sensibility of the ML solution to SNR has been assessed by comparing the RMSE of the estimates to the corresponding CRB given by Eq. (15). Repeated set of simulated targets have been generated to this purpose; for each group of trials different SNR have been exploited and results are summarized in Fig. 4. The trend of the RMSE of the estimates shows an asymptotic behavior with about $20 \mathrm{~dB}$ the inferior bound for which the estimation behaves approximately as $\mathrm{SNR} \rightarrow \infty$ (really, as the outcomes resulted noisy due to the relatively low number of trials, the measures have been fitted on a polynomial curve for a more readability of the plot). The spotlight processing provides superior performances compared to any possible stripmap-domain method, since the error of the estimates is below the CRB of the strimap estimation algorithms (i.e., the blue dotted line).

Finally, the impact of the not-ideality of point targets has been accounted for. The RMSE measures have been collected using spotlight processing ( $5 \times$ PRF) but making a simple sum in Eq. (13) instead of a weighted sum (i.e., supposing $\left.I_{p}=1, \forall p\right)$. The RMSE as a function of SNR is plotted in green in Fig. 4. Clearly the effect of the parameter defined in Eq. (12) produces more accurate results, further reducing the RMSE, at least for targets with $\mathrm{SNR}<15 \mathrm{~dB}$.

The algorithm has then been finally tested for several images taken from the Cosmo dataset. Fig. 5 shows one of these acquisitions out of about 30 taken on the same region, representing a suburban area 


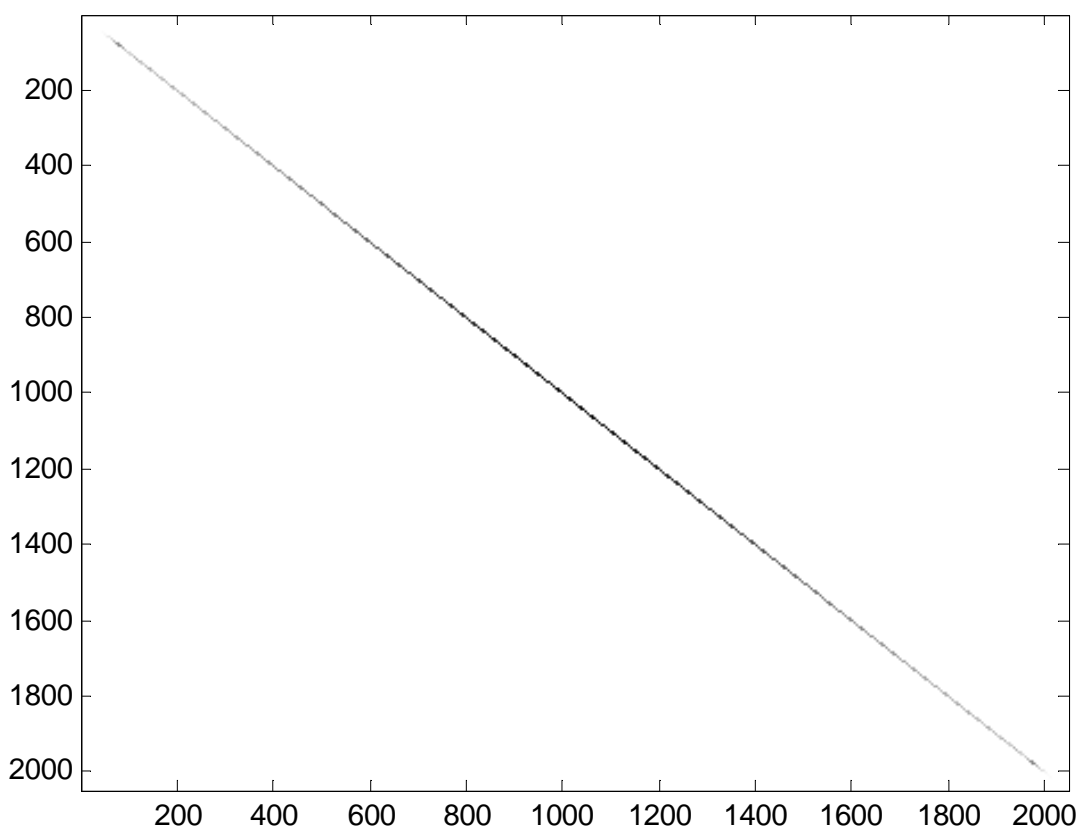

(a)

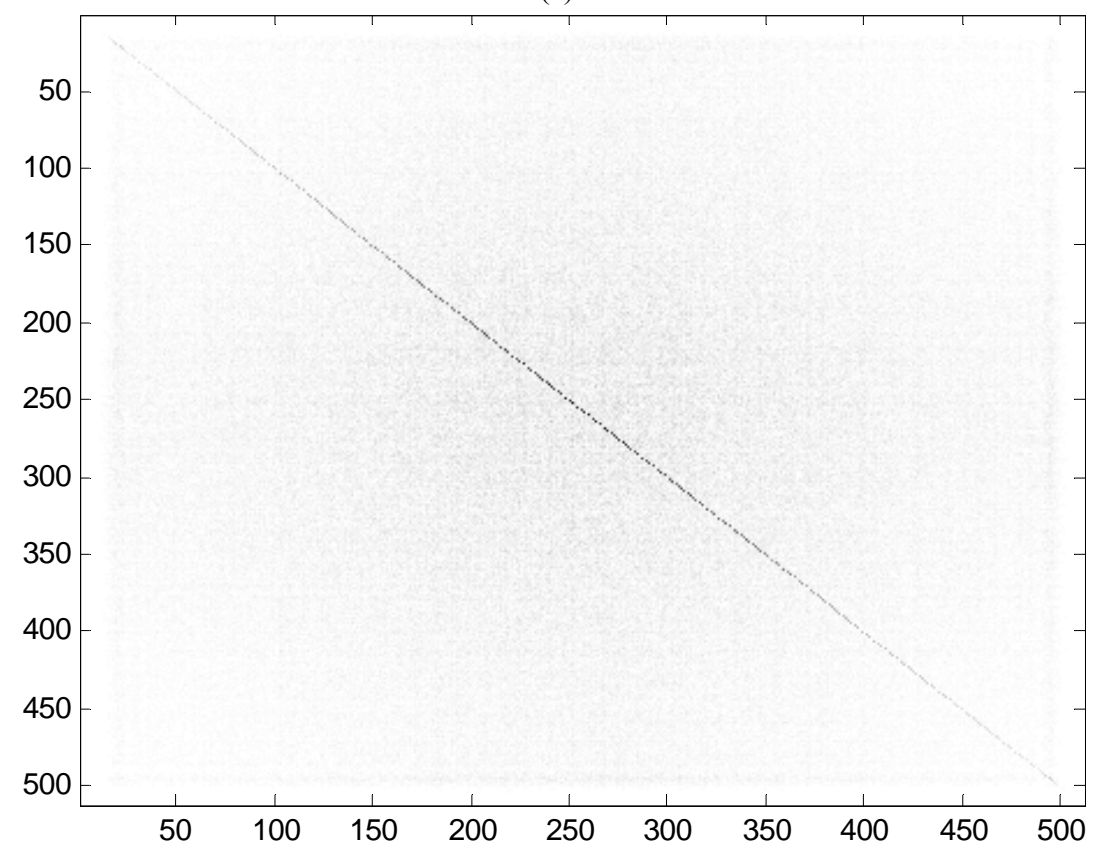

(b)

Fig. 2 Image plot of the covariance matrix. (a) covariance model computed as in (A3); (b) sample covariance matrix achieved averaging 128 targets spectra with average $\mathrm{SNR}=15 \mathrm{~dB}$ (stripmap processing applied).

located in North of Milan, Italy. On this area we expect to find a good number of point scatterers sufficiently isolated from other strong reflectors, for example little hangars, electricity poles, natural dihedral (two narrow orthogonal walls) and so on [18].

A set of 128 point targets has been selected for the estimation, with an average SNR of $15.1 \mathrm{~dB}$ and an average $I_{p}$ of 0.61 . It can be noticed that targets are preferably located within or close to urban areas; presumably man-made objects match better to the required point-shape criteria than natural targets. Target selection followed guidelines reported in Refs. [15-16]. 


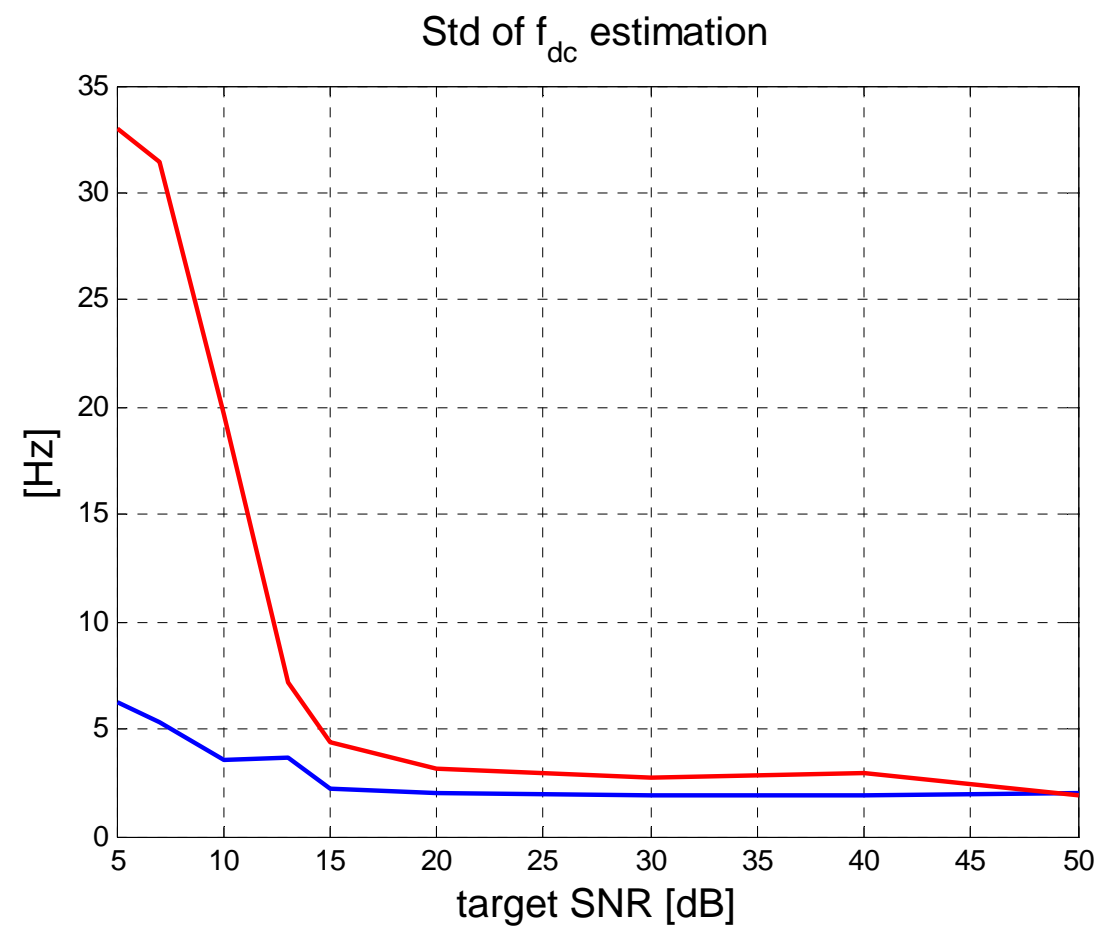

Fig. 3 Root mean square error of the DC estimate achieved by using simulated set of targets with various SNR. Red line: Eq. (14) solved by using the average of the targets spectra for $A_{a n t}(\bullet)$; blue line: Eq. (14) solved by using the antenna ideal model $\left(\operatorname{sinc}^{2}\right)$ in Eq. (14).

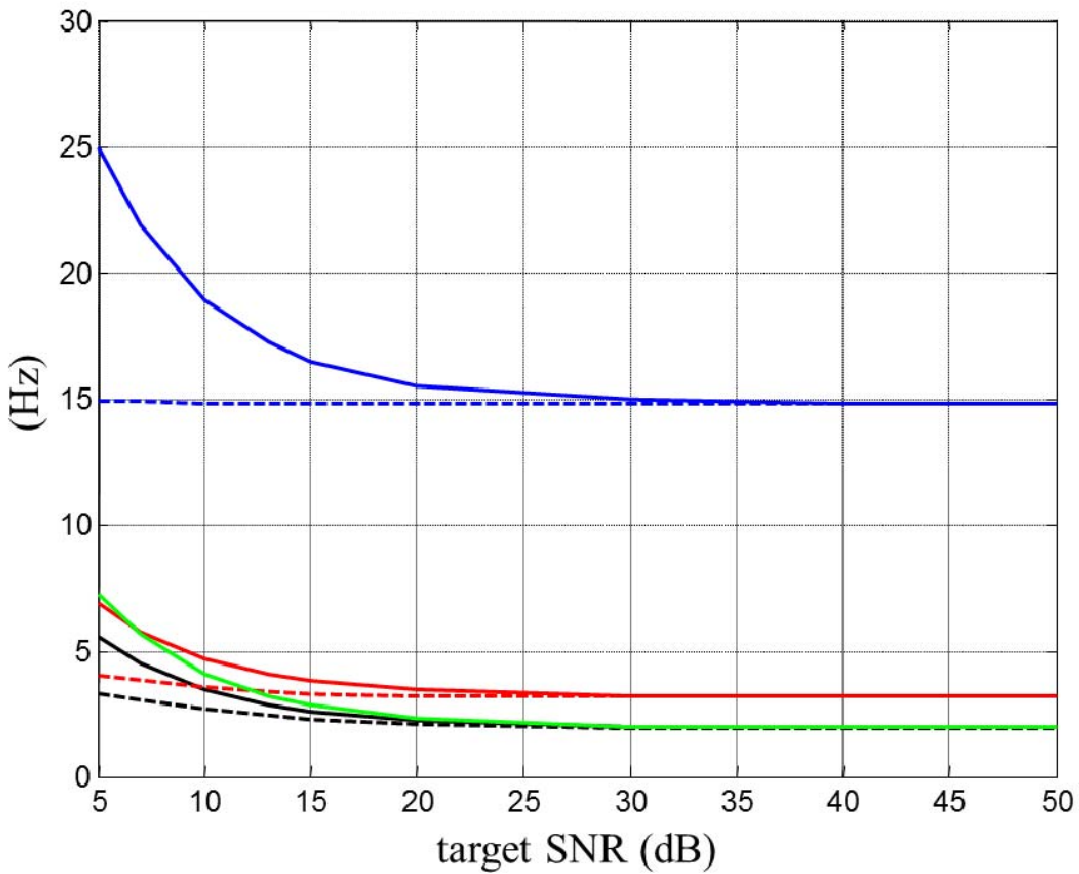

Fig. 4 Fitted RMSE (solid lines) compared with the Cramer Rao bound (dotted lines) for the estimate of $U$ vs. SNR. A set of 64 simulated targets has been generated in 32 different trials (averaged). Blue lines: $1 \times$ (i.e. stripmap) focusing; red lines: focusing at $3 \times$; black lines: focusing at $5 \times$. The green curve is the RMSE of a $5 \times$ estimate when $I_{p}=1$ in Eq. (14). For all the other cases the mean value of $I_{p}$ is 0.9 . 


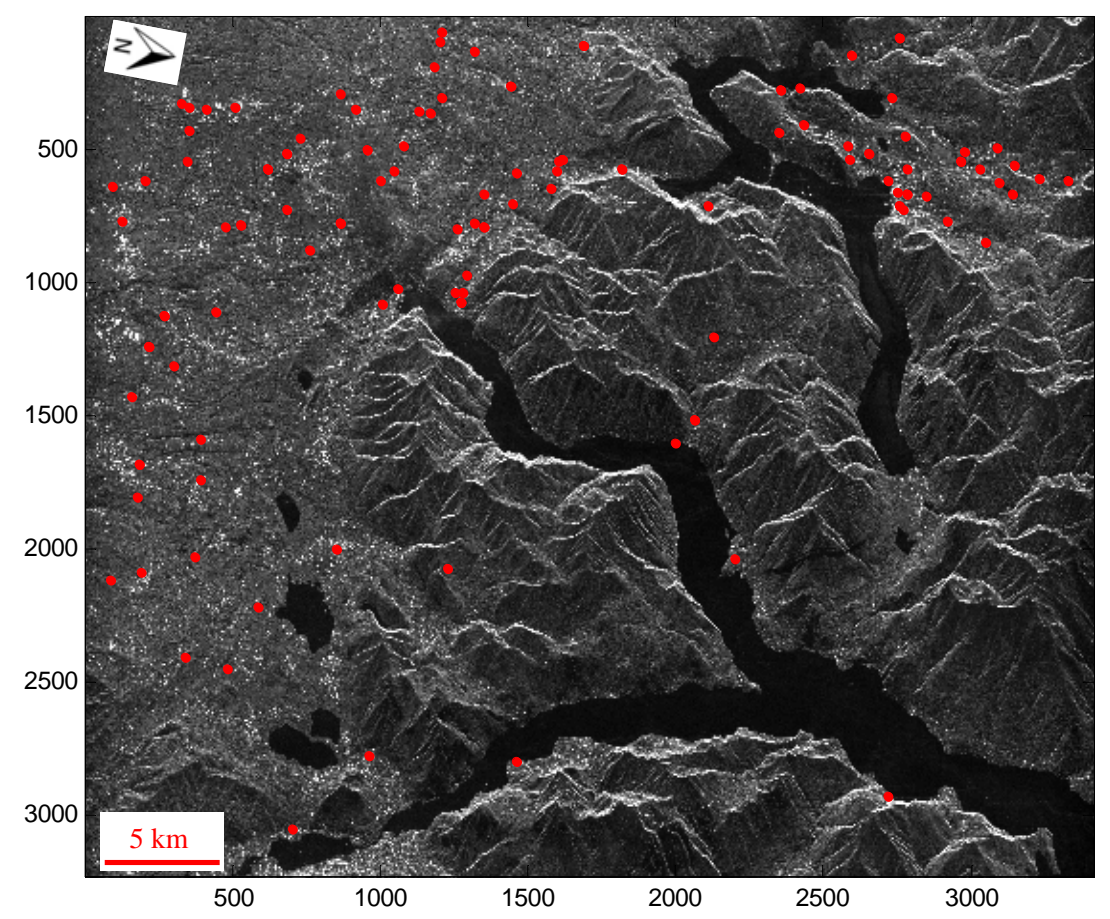

Fig. 5 Multilook image of the area at north of Milan, Italy and, in red, the targets selected for the DC estimation. Dataset acquired by Cosmo SkyMed 1 at $3^{\text {rd }}$ Sept. 2008 05:16:06. Image resolution is about $30 \mathrm{~m}$. Azimuth is the horizontal axis, slant range the vertical axis. Direction of flight is towards right, sensor view is from top to bottom.

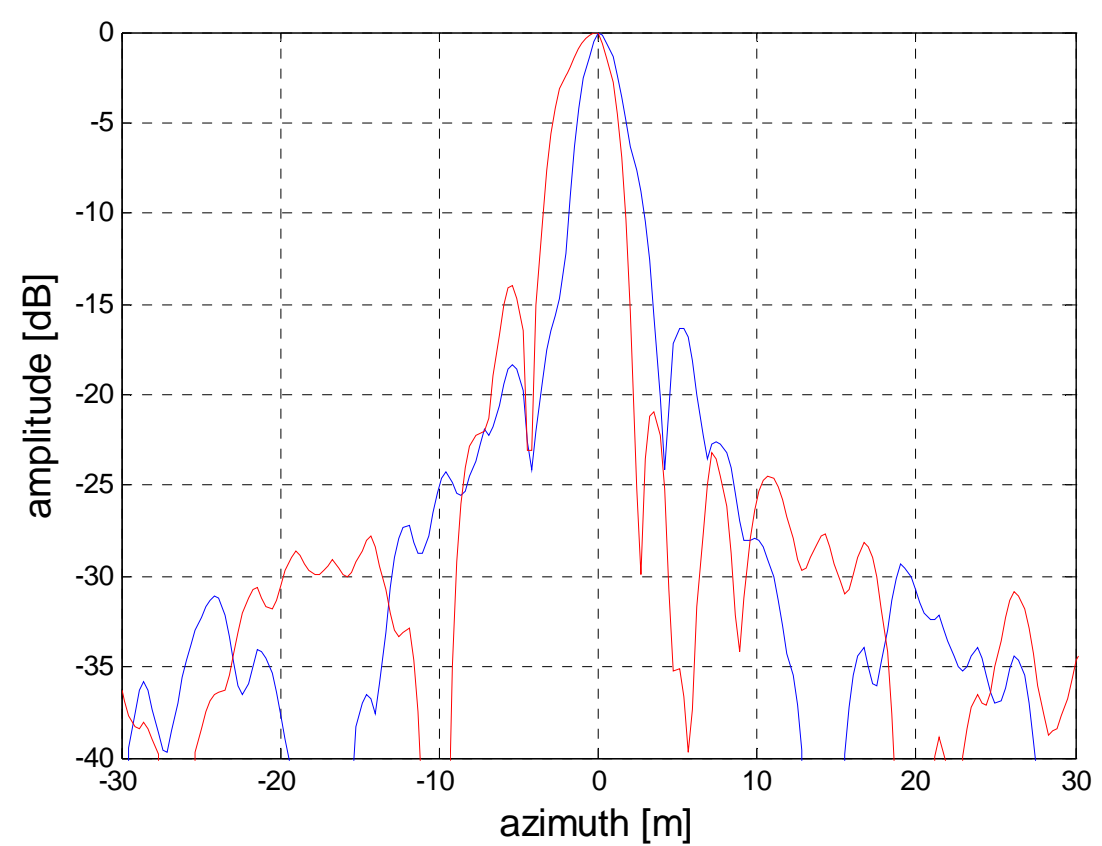

Fig. 6 Azimuth cut of a real point target in the Cosmo image before residual centroid correction (red curve) and after correction (blue curve). Target SNR is $23.8 \mathrm{~dB}$ and $I_{p} \simeq 0.8$. The $-3 \mathrm{~dB}$ lobe width resolution, the PSLR and the ISLR before and after centroid correction amount to, respectively, $3.260 \mathrm{~m}$ vs. $2.330 \mathrm{~m}, 13.95 \mathrm{~dB}$ vs. $16.30 \mathrm{~dB}, 13.03 \mathrm{~dB}$ vs. $9.79 \mathrm{~dB}$. 
Table 2 Targets performances before/after correction of $\hat{v}$. The percentiles refer to the distribution of the index $I_{p}$.

\begin{tabular}{llll}
\hline & $-3 \mathrm{~dB}$ resol $(\mathrm{m})$ & PSLR $(\mathrm{dB})$ & ISLR $(\mathrm{dB})$ \\
\hline 128 targets & 3.438 & 13.53 & 11.43 \\
128 targets $(v$ corrected $)$ & 3.087 & 13.57 & 10.47 \\
$10^{\circ}$ pecent $(115$ targets) & 3.486 & 13.40 & 11.48 \\
$10^{\circ}$ percent $(v$ corrected) & 3.109 & 13.46 & 10.46 \\
$50^{\circ}$ pecent $(64$ targets) & 3.629 & 12.68 & 11.42 \\
$50^{\circ}$ percent $(v$ corrected $)$ & 3.162 & 12.70 & 10.18 \\
$85^{\circ}$ pecent $(19$ targets $)$ & 3.864 & 12.88 & 11.11 \\
$85^{\circ}$ percent $(v$ corrected $)$ & 2.989 & 12.95 & 9.70 \\
\hline
\end{tabular}

Targets have been processed at $5 \times \mathrm{PRF}$ and estimation of $v$ has been achieved by using Eq. (14). After estimation, the correct $f_{d c}$ has been exploited for a new stripmap azimuth focusing. A better accuracy in determination of DC affects the final image resolution and the signal-to-ambiguity ratio: For strong and isolated scatterers these properties reflect in the target resolution and PSLR (peak to sidelobe ratio), which both must increase (sharper and delta-like peaks are expected) and in the ISLR (integrated to sidelobe ratio), which must decrease (more energy in the sidelobes).

An example of the azimuth cut of such a target is shown in Fig. 6. The improvement of the resolution as well as of the PSLR can be appreciated also visually. Performance in terms of resolution, PSLR and ISLR have been collected in two cases, i.e., before and after centroid correction in the stripmap focusing. Moreover, they have been measured on the full set of targets and on a restricted set; the restriction has been decided by tightening the level of the threshold for $I_{p}$ and discarding the targets under such threshold (i.e., keeping the best targets). Results in Table 2 show a progressive improvement of the parameters as the threshold tightens. The overall average improvement resulted of about $12 \%$ for the resolution, of $1 \%$ for the PSLR and $10 \%$ for the ISLR.

\section{Conclusions}

In this paper a method to estimate the DC for stripmap SAR data has been presented. The algorithm is based on an enhanced resolution processing, a proper selection of high quality targets and the ML estimation of the centroid as a parameter in a statistical model of the targets azimuth spectra. It has been shown that the algorithm is robust and accurate: estimation exhibits error close to the theoretical CRB even at moderate SNR (15 dB in X-band) and using a reasonable number of point targets (about one hundred).

Previous analysis on different land coverages in $\mathrm{X}$-band has shown that a good distribution of point targets, suitable for centroid estimation, is expected in urban, sub-urban and cropped areas. Although the targets spectral shape is not estimated, the model is able to provide accurate $\mathrm{DC}$, at least by exploiting the very point-shaped targets whenever present in few number. Estimation of target spectral signature in different land coverage conditions may be the possible extension of the method in a future work.

\section{Acknowledgments}

This work has been partly supported in the framework of ASI AO-1080, under the conditions as in ASI document DC-OST-2009-116.

\section{References}

[1] Doviak, R. J., Zrnic, D. S., and Sirmans, D. S. 1979. "Doppler Weather Radar." In Proceedings of the IEEE, 1522-53.

[2] Ruegg, M., Meier, E., and Nuesch, D. 2007. "Capabilities of Dual-Frequency Millimeter Wave SAR with Monopulse Processing for Ground Moving Target Indication." Geoscience and Remote Sensing, IEEE Transactions 45 (3): 539-53.

[3] Eldhuset, K. 1996. “Accurate Attitude Estimation Using 
ERS-1 SAR Raw Data.” International Journal of Remote Sensing 17 (14): 2827-44.

[4] Madsen, S. N. 1999. "Estimating the Doppler Centroid of SAR Data." IEEE Trans. Aerosp. Electron. Syst. 25: $135-40$.

[5] Bamler, R. 1991. "Doppler Frequency Estimation and the Cramer-Rao Bound." IEEE Trans. Geosci. Remote Sens. 29: 385-90.

[6] Cumming, I. G. 2004. "A Spatially Selective Approach to Doppler Estimation for Frame-Based Satellite SAR Processing." IEEE Trans. Geosci. Remote Sens. 42: 1135.

[7] Cumming, I. G., Kavangh, P. F., and Ito, M. R. 1986. "Resolving the Doppler Ambiguity for Spaceborne Synthetic Aperture Radar." In Proceedings of Geoscience and Remote Sensing Symposium, 1649-53.

[8] Bamler, R., and Runge, H. 1991. "PRF-Ambiguity Resolving by Wavelength Diversity." Geoscience and Remote Sensing, IEEE Transactions 29: 997-1003.

[9] Guccione, P., Cafforio, C., and Monti Guarnieri, A. 2004. "Doppler Centroid Estimation for ScanSAR Data." Geoscience and Remote Sensing, IEEE Transactions 42 (1): 14-23.

[10] Monti Guarnieri, A., and D'Aria, D. 2003. "Wide-Angle Azimuth Antenna Pattern Estimation in SAR Images." In Proceedings of IGARSS 2003, 3105-7.

[11] Prati, C., Monti Guarnieri, A., and Rocca, F. 1991. "Spot Mode SAR Focusing with the W-K Technique." In Proceedings of Geoscience and Remote Sensing
Symposium, IGARSS '91, 631-4.

[12] Guccione, P., Zonno, M., Mascolo, L., and D'Introno, S. 2014. "Persistent Point Scatterers Statistical Analysis for X-band SAR Data: The Cosmo SkyMed Case Study." International Journal of Remote Sensing 35 (1): 127-48.

[13] Guccione, P., and Monti Guarnieri, A. 2011. "ML Antenna Pattern Shape and Pointing Estimation in Synthetic Aperture Radar.” In 31st EARSeL Symposium Proceedings 2011.

[14] Carrara, W. G., Goodman, R. S., and Majewski, R. M. 1995. Spotlight Synthetic Aperture Radar. Artech House.

[15] Guccione, P., Monti Guarnieri, A., and Zonno, M. 2012. "Azimuth Antenna Maximum Likelihood Estimation by Persistent Point Scatterers in SAR images." Geoscience and Remote Sensing, IEEE Transactions 99: 1-10.

[16] Guccione, P., Monti Guarnieri, A., Giudici, D., and Tebaldini, S. 2012. "Long Term Relative Radiometric Calibration and Antenna Pointing Estimation by Natural Targets." IEEE Trans. Geosci. Remote Sensing 51 (8): 4388-96.

[17] Monti Guarnieri, A, Tebaldini, S., D'Aria, D., and Ferretti, A. 2010. "SAR Calibration Aided by Permanent Scatterer." Geoscience and Remote Sensing, IEEE Transactions 48: 2076-86.

[18] Perissin, D., and Ferretti, A. 2007. "Urban-Target Recognition by Means of Repeated Spaceborne SAR Image." Geoscience and Remote Sensing, IEEE Transactions 45: 4043-58.

\section{Appendix 1}

\section{A.1 Azimuth Spectrum for the End-to-end SAR System}

Taking the model in Section 2, the azimuth spectrum of the SAR data is the Fourier transform of Eq. (2) (dependence from distance $r$ is no longer represented for narrow strips):

$$
\begin{aligned}
& Z(f)=\mathfrak{J}\{z(t)\}=H_{s a r}(f) S(f)+N(f)= \\
& A_{a n t}\left(f-f_{d c}\right)|S(f)| \exp \left(-j \Phi\left(f ; f_{d c}\right)\right)+N(f)
\end{aligned}
$$

with $H_{\text {sar }}(f)=\mathfrak{J}\left\{h_{\text {sar }}(t)\right\}$ and being $\left|H_{\text {sar }}(f)\right|=A_{a n t}\left(f-f_{d c}\right)$ for the phase-matched filtering. The effect of the antenna shape in time domain is a function of the sensor-target angle-of-view $\psi=v t / r$, that is, under the narrow-band hypothesis, proportional to the Doppler frequency $f: \psi \simeq-\lambda f / 2 v$. For this reason the antenna amplitude shape is the same both in time and frequency domains. The spectrum phase $\Phi$ is the Fourier transform of the focused target phase and accounts for contributions coming from the target features, the overall target-to-sensor two way optical path, the atmospheric screen and noise [17]. Finally, the Fourier transform of the complex reflectivity $S(f)$ is related to the electromagnetic behavior of a real target (both point and distributed) under different acquisition geometries, frequencies and polarization. The shape of $S(f)$ in the main lobe allows to infer information on the real point target extension, while the phase may catch detail on the nature of the target [18].

\section{A.2 The Covariance Matrix}

According to the notation in Section 3.1, the covariance matrix of $\mathbf{z}$ can be computed as: 


$$
\begin{aligned}
& C_{\mathbf{z}}=E\left[(\mathbf{z}-E[\mathbf{z}])(\mathbf{z}-E[\mathbf{z}])^{*}\right]= \\
& \sigma_{s}^{2} \cdot \mathbf{h} \mathbf{I h}^{*}+\sigma_{n}^{2} \cdot \mathbf{h}_{f} \mathbf{I h}_{f}^{*}
\end{aligned}
$$

with $\mathbf{h}_{f}$ the N-column array of the sampled version of $h_{f}(t), \mathbf{I}$ the identity matrix and $[\bullet]^{*}$ the Hermitian operator. In this formulation, distributed scatterer and thermal noise are supposed uncorrelated each other; furthermore, the contribution of the point target $\mathbf{p}$ provides a systematically not-null mean in the data source $\mathbf{s}$ and in the image data $\mathbf{z}$.

In the vector formulation, the Discrete Fourier Transform can be represented by a matrix-vector multiplication: $\mathbf{Z}=\mathbf{W}^{*} \mathbf{Z}$ with W the orthogonal matrix including the Fourier kernel for all the input/output indexes. Using this notation, it can be shown that the covariance matrix of $\mathbf{Z}$ is approximately diagonal since the spectral components of a colored process (as for example the reflectivity source passed through the end-to-end SAR system) are uncorrelated, with the specific frequency components having different variance:

$$
\begin{aligned}
& C=E\left[(\mathbf{Z}-E[\mathbf{Z}])(\mathbf{Z}-E[\mathbf{Z}])^{*}\right] \\
& =\sigma_{s}^{2} \cdot \mathbf{W}^{*} \mathbf{h h}^{*} \mathbf{W}+\sigma_{n}^{2} \cdot \mathbf{W}^{*} \mathbf{h}_{f} \mathbf{h}_{f}^{*} \mathbf{W} \\
& \simeq \sigma_{s}^{2} \cdot \mathbf{H H}^{*}+\sigma_{n}^{2} \cdot \mathbf{I}
\end{aligned}
$$

where $\mathbf{H}=\mathbf{W}^{*} \mathbf{h}$ is the (matrix) transfer function of $\mathbf{h}$. In principle, the image noise for SAR data is not uncorrelated and the covariance matrix not perfectly diagonal; however, the approximation in Eq. (A2) is reasonable for strong targets, i.e., $\sigma_{s}^{2} / \sigma_{n}^{2} \gg 1$ and by assuming the bandwidth of the focusing operator $\mathbf{H}_{f}$ to be constant in the non-ambiguous domain. This assumption is experimentally justified in Section IV.

With the previous assumptions, the covariance matrix can be finally written as:

$$
C \simeq \operatorname{diag}\left[\sigma_{s}^{2}\left|H\left(f_{m}\right)\right|^{2}+\sigma_{n}^{2}\right], \quad m=1, \ldots, M
$$

which is the formulation in Eq. (4). 